\title{
CONCEPT OF ORGANIC PHOTOVOLTAICS: OPERATIONAL PRINCIPLES AND MATERIALS
}

\author{
Július CIRÁK \\ Institute of Nuclear and Physical Engineering, Faculty of Electrical Engineering and Information Technology, \\ Slovak University of Technology in Bratislava, Ilkovičova 3, 81219 Bratislava, Slovak Republic, tel. +421 260291138 , \\ e-mail: julius.cirak@stuba.sk
}

\begin{abstract}
Organic molecules possessing a $\pi$-conjugated (hetero)aromatic backbone are capable of transporting charge and interact efficiently with light. Therefore, these systems can act as semiconductors in opto-electronic devices similar to inorganic materials. However, organic chemistry offers tools for tailoring materials' functional properties via modifications of the molecular units, opening new possibilities for inexpensive device manufacturing. In this presentation, we discuss exploitation of such organic molecular systems in photovoltaics; the operation, advantages, and limitations of molecular donor-acceptor heterojunction structures.
\end{abstract}

Keywords: Organic photovoltaics, donor - acceptor heterojunctions, small molecules, organic semiconductors

\section{INTRODUCTION}

Classical photovoltaics (PV) has an unfavourable cost structure compared to alternative renewable energy technologies; nevertheless the continued high interest in this technology originates from the fact that photovoltaics is the only true portable and renewable source of energy available today. The Sun is the largest energy source, which can provide for the Earth the power of $124 \times 10^{15} \mathrm{~W}$, more than 8000 times of the total worldwide consumption. The direct conversion of solar radiation into electricity is a simple and clean way to harness such a vast energy source. The photovoltaic effect requires active semiconducting materials, and currently inorganic solar cell based on silicon is the dominant technology. It exhibits good performance in power conversion efficiency (PCE) and lifetime. However, the high production and installation cost of silicon solar cells limit their widespread use to provide a large-fraction of our electricity. Due to this the thought of PV elements based on thin plastic carriers, manufactured by printing and coating techniques from reel to reel, and packaged by lamination techniques is not only intriguing but highly attractive from a cost viewpoint [1]. In order to fulfil these conditions, high volume production technologies for large area coating must be applied to a low cost material class. Solution processable organic and inorganic semiconductors have a high potential to fulfil these requirements [2]. Flexible chemical tailoring allows the design of organic semiconductors with desired properties, and printing or coating techniques like screen, inkjet, offset or flexo printing are being established for semiconducting polymers today, driven by display or general electronic demands. Altogether, organic PV has many attractive features [3], amongst them:

- the potential to be flexible and semitransparent,

- potential to be manufactured in a continuous printing process,

- large area coating,

- easy integration in different devices,
- significant cost reduction compared to traditional solutions,

- substantial ecological and economic advantages.

Furthermore, the physical properties of organic solar cells, such as absorption spectrum and charge transport property, can be tailored by chemical synthesis. The thickness of an active layer can be very small (ca.100 nm) due to their high absorption coefficient within their absorption range so that organic solar cell can be semitransparent and the color can be tuned. The lightweight and mechanical flexibility of polymer materials enable some specific applications of organic solar cells, such as in portable devices.

The donor/acceptor concept pioneered by Tang [4] in 1986 and bulk-heterojunction (BHJ) design developed by Yu et al. [5] in 1995 led to the active pursuit of polymer solar cells. The solution processed BHJ solar cells are easy to prepare and present a high density of heterojunction interfaces that enables the efficient exciton dissociation and charge generation over the whole active layer, which made a substantial increase in power conversion efficiency of the device. These two progresses established the solution processed BHJ solar cells as the most promising technology for organic solar cells. Gradual progress in the solar cell performance is underway based on this architecture. PCE evolved from 1 $\%$ in 1995, to $5 \%$ in 2005 and almost $10 \%$ most recently [6].

Despite the mentioned advantages and recent technology advances, so far polymer solar cells still lag behind inorganic counterparts in terms of PCE and life times. Solar cells with PCE over $10 \%$ are necessary for widespread applications. After an exhaustive effort in morphology control, electrode modification, the introduction of dielectric layers, and the utilization of the plasmonic effect, it is clear that the most important limiting factor for the further improvement on the photovoltaic performance is the material in active layers, especially the polymer donor material [7]. 


\section{ORGANIC PHOTOVOLTAICS - CONCEPT}

A PV cell is a specialized semiconductor diode that converts visible light into direct electric current (DC). Some PV cells can also convert infrared (IR) or ultraviolet (UV) radiation into DC. A common characteristic of both the small molecules and polymers used in organic photovoltaics (OPV) is that they all have extended conjugated systems (see Fig. 1). A conjugated system is formed where carbon atoms covalently bind with alternating single and double bonds. These hydrocarbons electrons $\mathrm{p}_{\mathrm{z}}$ orbitals delocalize and form a delocalized bonding $\pi$ orbital with a $\pi^{*}$ antibonding orbital. The delocalized $\pi$ orbital is the highest occupied molecular orbital (HOMO), and the $\pi^{*}$ orbital is the lowest unoccupied molecular orbital (LUMO). The separation between HOMO and LUMO is considered as the band gap of organic electronic materials. The band gap is typically in the range of $1-4 \mathrm{eV}$.

When these materials absorb a photon, an excited state is created and confined to a molecule or a region of a polymer chain [3]. The excited state can be regarded as an electron-hole pair bound together by electrostatic interactions, i.e. excitons. In PV cells, excitons are broken up into free electrons-hole pairs by effective fields. The effective field is set up by creating a heterojunction between two dissimilar materials. Effective fields break up excitons by causing the electron to fall from the conduction band of the absorber to the conduction band of the acceptor molecule. It is necessary that the acceptor material has a conduction band edge that is lower than that of the absorber material. consisting of a single polymeric semiconductor layer EQEs are typical below $1 \%$. A solution was only found in 1995, when several groups independently showed that the EQE could be enhanced by several orders of magnitude upon blending two materials with relative preferences for positive and negative charges [5,8]. The difference in electron affinities creates a driving force at the interface between the two materials which is strong enough to split photogenerated excitons. By blending the materials on a nanostructured scale (about $10 \mathrm{~nm}$ ), the interface is distributed throughout the whole device. Hence, all the photogenerated excitons are likely to find an interface and split before recombining if the charge transfer is significantly faster than the competing recombination channels, either radiatively or non-radiatively. The separated charges must then travel through the appropriate material towards the contacts. The concept of blending ptype semiconductors with n-type semiconductors has become popular under the name bulk heterojunction (BHJ) composites [9].

The EQE represents that fraction of photoexcitations which survive both charge separation and transport processes. Using the BHJ concept the EQE has been increased to over $80 \%$. These impressive values have been obtained for blends of conjugated polymers with fullerenes.

Solution processing may qualify polymeric photovoltaics as an alternative renewable energy source. Unfortunately, overall power conversion efficiencies (PCE) under the normalized solar conditions are low. One of the main reasons for this still somehow disappointingly low PCE despite the high EQE is the spectral mismatch of
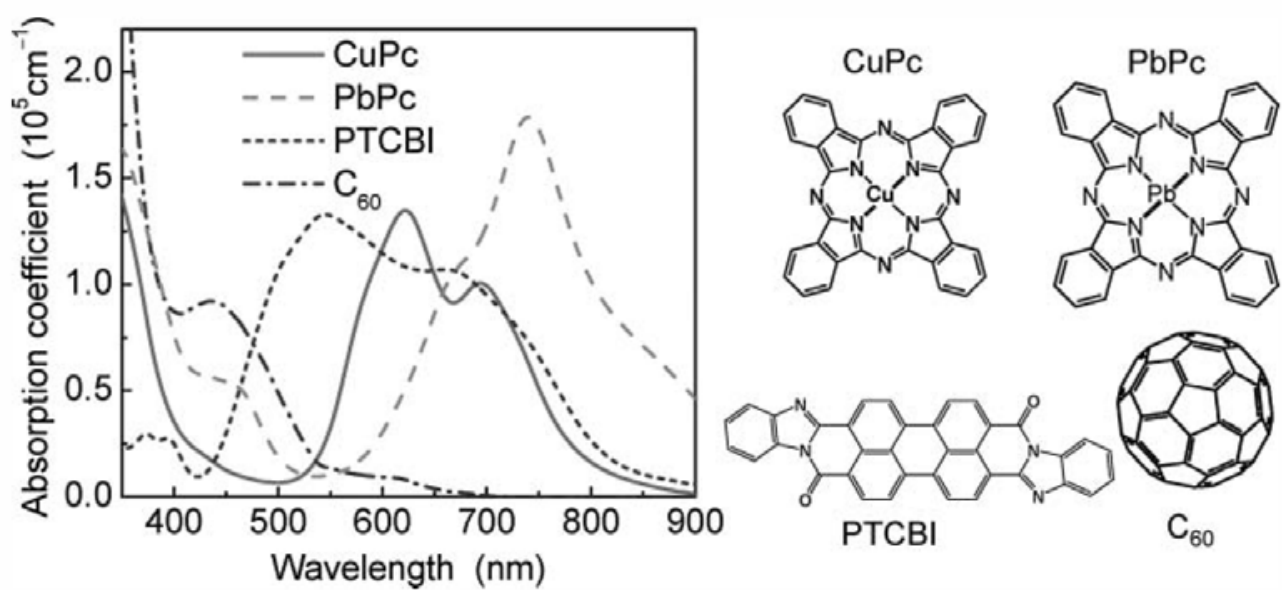

Fig. 1 Absorption spectra and molecular structures of four small molecular weight organic compounds used in photovoltaic cells. Here CuPc and PbPc are copper and lead phthalocyanines, respectively, PTCBI is 3,4,9,10-perylene tetracarboxylic bisbenzimidazle, and $\mathrm{C}_{60}$ is fullerene [12]

Most organic semiconductors are intrinsic semiconductors and the primary excitation is a Coulomb bound exciton. PV cells made of single organic semiconductors therefore achieve small power conversion efficiencies and low incident-photon-to current or external quantum efficiency (EQE). A high EQE does not guarantee good photovoltaic energy conversion, but it is a necessary prerequisite. For inorganic PV devices routinely EQEs approaching $100 \%$ are achieved. For organic PV devices the organic absorbers to the solar spectrum. Most of the organic semiconductors investigated today absorb in the visible range, while the Sun has its maximum photon density at around $700 \mathrm{~nm}$. Another problem is a certain intrinsic limited durability of organic compounds: when electrons are excited to higher orbitals, anti-binding states arise and the probability for decomposition of the compound increases. This is particularly true for n-type organic semiconductors. 
Finally, effective light harvesting in a blend PV device demands efficient charge separation and transport. First, the energy bands of the two materials have to cascade in order to guarantee charge generation after photoexcitation (Fig. 2a). Second, each material must provide a continuous path for the transport of separated charge to the contacts (Fig. 2b). This demands a highly complex morphology between the two compounds with interconnected domains in the nanometer scale to prevent trapping and more ordered regions in the $100 \mathrm{~nm}$ scale to guarantee efficient transport channels.

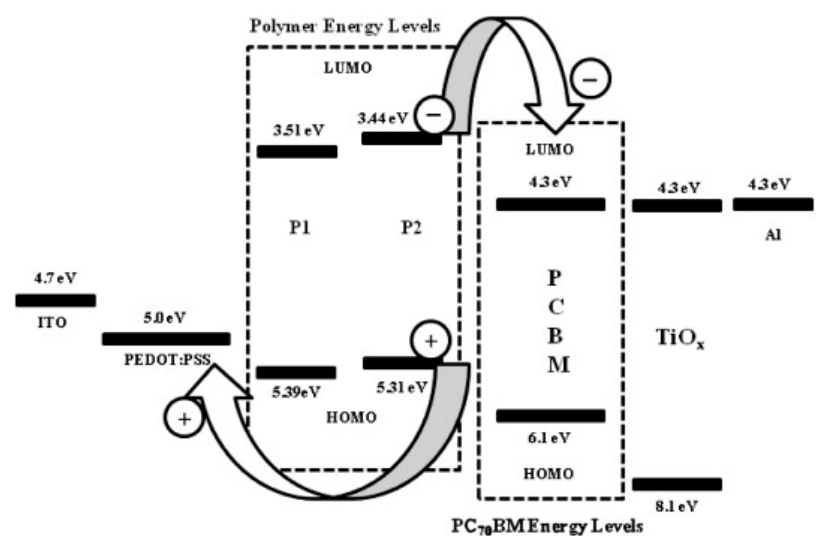

Fig. 2a Energy bands of the two materials in a bulk heterojunction solar cell [3]

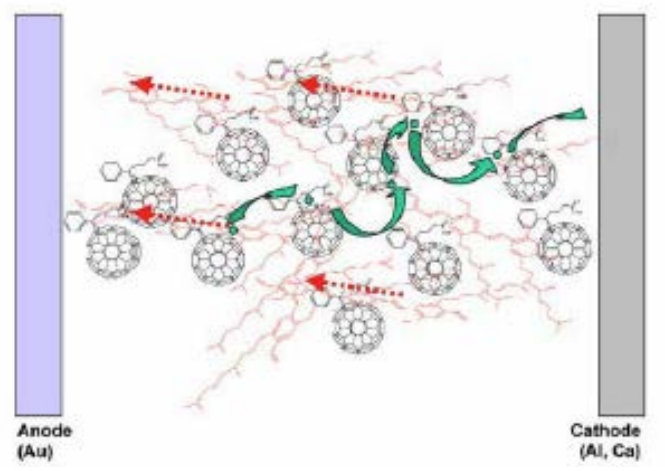

Fig. 2b Schematic drawing for charge transport in bulk heterojunction composites [3]

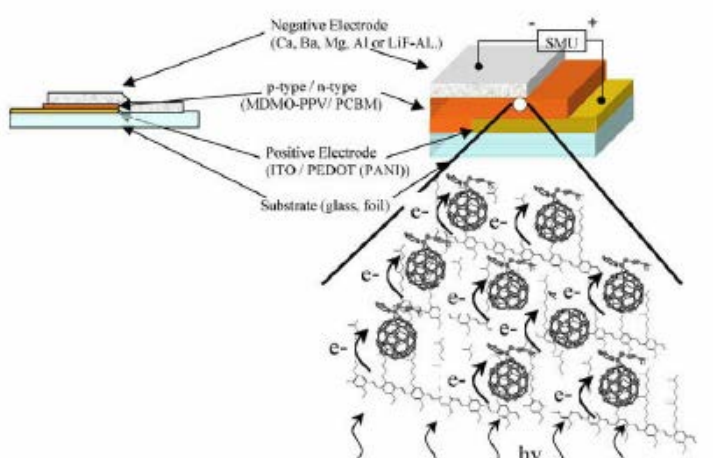

Fig. 2c Schematic drawing of the thin film solar cells

\section{MATERIALS FOR BHJ ORGANIC SOLAR CELLS}

There are two components in the BHJ structure, the donor and the acceptor. The selection of the materials for both components is very important for solar cell performance. Owing to their strong absorbing ability, good film-forming ability, semiconducting polymers are good candidates as a donor component. A potential drawback is that conjugated polymers exhibit a broad distribution in molecular weight, which is easy to cause purification difficulty and poor reproducibility due to batch-to-batch difference. Small conjugated molecules offer the advantage of mono-dispersity and easy purification, so they are actively searched for solar cell applications. However, the film forming ability from solution and the charge mobility in the blend structure of small molecules, are not as good as of polymers.

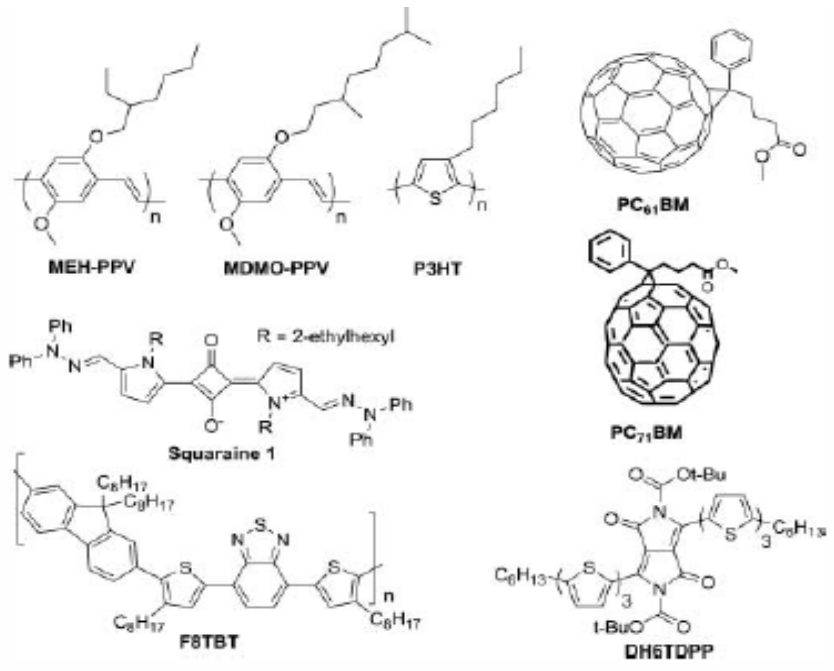

Fig. 3 Structures of widely used conjugated polymers and fullerene derivatives

As acceptor components fullerene derivatives are found to be the best candidates due to their high electron affinity and superior electron mobility [11]. Especially, the 3D structure of fullerene offers unique packing ability in blend, which can form the electron transport channels efficiently. The original fullerenes do not have enough solubility in organic solvents, so fullerene derivatives with solubilizing groups are usually used. Introduction of the functional group usually improves the miscibility with the donor components, but with a small impact on the electronic properties of fullerene. As a result, fullerene derivatives BHJ structure can provide the advantages of efficient exciton dissociation by intermixing the donor/acceptor phases well enough and efficient extraction of the separated charges from the interface by high electron mobility. The weakness of fullerene derivatives as acceptor materials in BHJ solar cells lies in their weak absorption in visible region $\left(\mathrm{C}_{60}\right)$ and expensive materials (like $\mathrm{C}_{70}$ ). 


\section{DEVICE CHARACTERIZATION}

In this section the parameters used to characterize the performance of PV devices will be described briefly. The devices are generally characterized by the shortcircuit current $\left(J_{S C}\right)$, the open-circuit voltage $\left(V_{O C}\right)$ and the fill factor (FF). The fill factor of a device is defined as the ration between the maximum power delivered to an external circuit and the potential power:

$F F=P_{m} / J_{S C} V_{O C}=J_{m} V_{m} / J_{S C} V_{O C}$

The fill factor is the ratio of the darkly shaded to lightly shaded regions in Fig. 4.

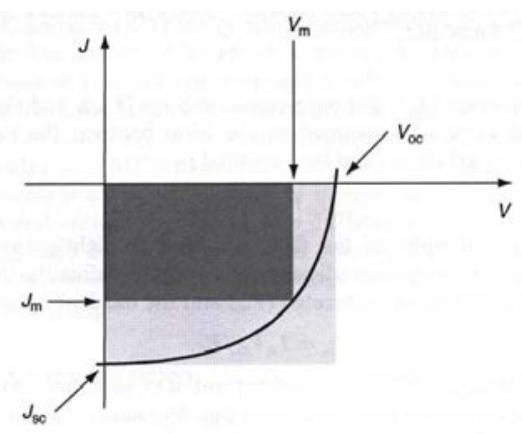

The external quantum efficiency (EQE), also known as the incident photon conversion efficiency, is given by the number of electrons generated per incident photon:

$E Q E=\frac{n_{e}}{n_{p h}}=\frac{I_{S C}}{P_{O}} \frac{h c}{\lambda e}$,

where PO is the incident optical power, $h$ is Planck's constant, $c$ is the speed of light, $\lambda$ is the wavelength of light, and $e$ is the charge of electron. This generally follows the absorption spectrum of the material constituting the organic PV cell. The internal quantum efficiency (IQE), also known as the photocurrent action spectrum, is given by the ratio of the photocurrent to the absorbed photon flux.

\section{CONCLUSIONS}

Since the discovery in 1995, polymer/fullerene BHJ solar cells constitute a very popular research area, and a better understanding of the operating mechanism and factors that affect the solar cell performance have been obtained. However, the observed efficiency in polymer solar cells, especially in large solar panels is still lower than the corresponding commercial inorganic systems, which limits their large scale applications.

It has been realized that the ideal polymer in BHJ structure should exhibit a broad absorption with a high coefficient in the solar spectrum to ensure effective harvesting of solar photons. In addition, the polymer should have high hole mobility for charge transport. To maximize the effective charge carriers extraction, the hole mobility of the polymer should be balanced with the electron mobility of the fullerene acceptor. The polymer is required to have suitable energy levels matching the fullerene. The polymer should also have a low-lying HOMO energy level to provide a large $V_{O C}$ and suitable
LUMO energy level to provide enough offset for charge separation. Besides these optical and electronic properties, the polymer should have appropriate compatibility with fullerene to form effective bicontinuous interpenetrating network in nanoscale for charge separation and subsequent transport. A good example is the abovementioned P3HT/PCBM system, based on which the BHJ solar cells exhibited a PCE close to $8 \%$ [11].

Nevertheless, several challenges still exist in this area. First, new donor materials with better properties are still needed. Second, acceptor polymers that can rival fullerenes are essential to lower the cost of polymer solar cells. Third, a detailed understanding of the photochemical stability of the organic cells must be gained. The progress achieved so far in this area makes us to believe that solutions can be found and organic BHJ solar cells will have a bright future.

\section{ACKNOWLEDGEMENTS}

This publication originates from the project "National Centre for Research and Applications of Renewable Energy Sources” (IMTS 26240120028) of Operational program Research and Development financed by European fund of Regional Development and the work was supported by the Slovak Republic grant agency VEGA, project No. 1/0879/11.

\section{REFERENCES}

[1] WONG, W. S. - SALLEO, A. (Eds.): Flexible Electronics: Materials and Applications, Electronic Materials Science \& Technology, Springer Science + Business Media, 2009.

[2] SUN, S. S. - SARICIFTCI, N. S.: Organic Photovoltaics, CRC Press, New York, 2005.

[3] BRABEC, Ch. J.: Organic photovoltaics: technology and market, Solar Energy and Solar Cells, vol. 83, pp. 273 - 292, 2004.

[4] TANG, C. W.: Two-layer organic photovoltaic cell, Appl. Phys. Lett., vol. 48, pp. 183 - 185, 1986.

[5] YU, G. - GAO, J. - HUMMELEN, J. C. - WUDL, F. - HEEGER, A. J.: Polymer photovoltaic cells: Enhanced efficiencies via a network of internal donor-acceptor heterojunctions, Science, vol. 270, pp. 1789 - 1791. 1995.

[6] LEWIS, N. S.: Toward cost-effective solar energy use, Science, vol. 315, pp. 798 - 801, 2007.

[7] CHENG, Y. J. - YANG, S. H. - HSU, C. S.: Synthesis of conjugated polymers for organic solar cell applications, Chem. Rev., vol. 109, pp. 5868 5923, 2009.

[8] HALLS, J. J. M. - WALSH, C. A. - GREENHAM, N. C. - MARSEGLIA, E. A. - FREND, R. H. MORATTI, S. C. - HOLMES, A. B.: Efficient photodiodes from interpenetrating polymer networks, Nature, vol. 376, pp. 498 - 500, 1995.

[9] SARICIFTCI, N. S. - SMILOWITZ, L. - HEEGER, A. J. - WUDL, F.: Photoinduced Electron Transfer 
from a Conducting Polymer to Buckminsterfullerene, Received December 10, 2012, accepted January 15, 2013 Science, vol. 258, pp. 1474 - 1477, 1992.

[10] WALDAUF, C. - SCHILINSKI, P. - HAUCH, J. BRABEC, C. J.: Material and device concepts for organic photovoltaics: towards competitive efficiencies, Thin Solid Films, vol. 451 - 452, pp. $503-507,2004$.

[11] LIANG, Y. - LUPING, Y.: Development of semiconducting polymers for solar energy harvesting, Polymer Reviews, vol. 50, pp. 454 - 473, 2010.

[12] ZHENG, Y. - XUE, J.: Organic photovoltaic cells based on molecular donor - acceptor heterojunctions, Polymer Reviews, vol. 50, pp. 420 453, 2010.

\section{BIOGRAPHY}

Július Cirák was born on the 29th June 1953 in Likier. He graduated from the Faculty of Electrical Engineering, Slovak University of Technology, Bratislava, in 1976, and received his $\mathrm{CSc}(\mathrm{PhD})$ degree in experimental physics in 1981. He joined the Department of Physics at the same Faculty, as an Assistant (1982), Associate (1988) and Full Professor (2008). His research experience and interest include: organic molecular thin layer systems and nanostructures; technology, study of electrical and optical properties and applications for organic electronics. 